\title{
Blockchain Applications for Healthcare Data Management
}

\author{
Dimiter V. Dimitrov \\ Diavita Ltd., Varna, Bulgaria
}

Objectives: This pilot study aimed to provide an overview of the potential for blockchain technology in the healthcare system. The review covers technological topics from storing medical records in blockchains through patient personal data ownership and mobile apps for patient outreach. Methods: We performed a preliminary survey to fill the gap that exists between purely technically focused manuscripts about blockchains, on the one hand, and the literature that is mostly concerned with marketing discussions about their expected economic impact on the other hand. Results: The findings show that new digital platforms based on blockchains are emerging to enabling fast, simple, and seamless interaction between data providers, including patients themselves. Conclusions: We provide a conceptual understanding of the technical foundations of the potential for blockchain technology in healthcare, which is necessary to understand specific blockchain applications, evaluate business cases such as blockchain startups, or follow the discussion about its expected economic impacts.

Keywords: Health Information Interoperability, Information Storage and Retrieval, Genomics, Artificial Intelligence, mHealth

\section{Introduction}

A blockchain, by design and definition, is a particular type of database [1]. It is constructed as a to-read-only-once database. This means that blockchain databases are designed to

Submitted: December 18, 2018

Revised: January 11, 2019

Accepted: January 23, 2019

\section{Corresponding Author}

Dimiter V. Dimitrov

Diavita Ltd., 68 Br. Miladinovi str. G, Varna 9000, Bulgaria. Tel:+35987777-9937, E-mail: dimiter.v.dimitrov@gmail.com (https://orcid. org/0000-00003-3766-5850)

This is an Open Access article distributed under the terms of the Creative Commons Attribution Non-Commercial License (http://creativecommons.org/licenses/by$\mathrm{nc} / 4.0 /$ ) which permits unrestricted non-commercial use, distribution, and reproduction in any medium, provided the original work is properly cited.

(c) 2019 The Korean Society of Medical Informatics be only-ever-created, and not edited or deleted. Data stored in a blockchain decentralized ledger (computer file asset) is a transactional type of data that requires space of $1 \mathrm{kB}$ or less, and no one is able to access it as long as the owner holds the private keys. Moreover, the owner can use the InterPlanetary File System (IPFS) to access the data and transfer it from one computer to another much faster and more securely and economically in comparison to centralized databases [2].

The rationale for the use of blockchain technology in healthcare is the fact that maintenance of a typical healthcare information system involves various operations including but not limited to performing backup storage services, having recovery mechanisms in place, and ensuring up-to-date fields [3]. In a blockchain, data are distributed across the network, and there is no single point of failure leading to an inherent backup mechanism. Also, a single version of data is copied on every node of the blockchain. This reduces the volume of transactions that occur between information sys- 
Table 1. Blockchain companies for healthcare data management

\begin{tabular}{|c|c|c|c|}
\hline & \multicolumn{3}{|c|}{ Blockchain company } \\
\hline & Name & Country & Website \\
\hline \multirow[t]{3}{*}{ EMR data management } & PokitDoc & USA & http://pokitdoc.com \\
\hline & Gem & USA & http://enterprise.gem.co/health \\
\hline & YouBase & USA & http://www.youbase.io \\
\hline \multirow[t]{6}{*}{ EHR data management } & Medicalchain & USA & http://www.medicalchain.com \\
\hline & HealthWizz & USA & http://www.healthwizz.com \\
\hline & Curisium & USA & http://www.curisium.com \\
\hline & Hearthy & Spain & http://hearthy.co \\
\hline & Iryo & Slovenia & http://iryo.io \\
\hline & Robomed & Russia & http://www.robomed.io \\
\hline \multirow[t]{2}{*}{ PHR data management } & Medcredits & USA & https://medcredits.io \\
\hline & MyClinic & $\mathrm{UK}$ & https://myclinic.com \\
\hline \multirow[t]{4}{*}{ Point-of-care genomics } & Nebula Genomics & USA & http://www.nebula.org \\
\hline & Genomes.io & USA & http://www.genomes.io \\
\hline & TimiCoin & USA & http://www.timicoin.io \\
\hline & Shivom & Switzerland & http://shivom.io \\
\hline Oncology patients network & OncoPower & USA & http://oncopower.org \\
\hline \multirow[t]{3}{*}{ Pharma \& drug development } & Embleema & France & http://www.embleema.com \\
\hline & BlockPharma & France & http://www.blockpharma.com \\
\hline & Chronicled MediLedger & USA & http://www.mediledger.com \\
\hline
\end{tabular}

EMR: Electronic Medical Record, EHR: Electronic Health Record, PHR: Personal Health Record.

tems, thus reducing the burden on the healthcare ecosystem [4].

With the progress in electronic health-related data gathering, cloud healthcare data storage and patient data privacy protection regulations, new opportunities are opening for daily health data management, as well as for convenience for patients to access and share their own health data (Table 1). In this study, the available applications of blockchains in healthcare and cases of their use are reported in general.

\section{Case Description}

\section{Blockchain for Electronic Medical Record (EMR) Data Management}

The potential for the use of blockchain technology in hospitals has started to be tested in several pilot projects globally. Last year in the United States, Booz Allen Hamilton Consulting developed and implemented a blockchain-based pilot platform designed to help the Food and Drug Administration's Office of Translational Sciences explore how to use the technology for healthcare data management (Figure 1). The pilot project is currently being implemented at four major hospitals; it is using Ethereum to manage data access via virtual private networks. The project is built on the IPFS to utilize encryption and reduce data duplication via off-chain cloud components with cryptographic algorithms to create user sharing [6].

\section{Blockchain and Healthcare Data Protection}

In Europe the relationship between blockchains and the General Data Protection Regulation (GDPR) is somewhat controversial. On one hand, blockchains seem to represent a good alignment with GDPR (when it comes to data portability, as an example, or consent management, data traceability and lawful access auditability). On the other hand, various issues can be identified (when it comes to right to be forgotten, but also when the technical implementation through smart contracts might weaken the actual control over data, through automatic execution). One option to tackle this issue is 'dynamic consent management', which is fully in line with the GDPR provision regarding consent [7]. In addition, it is considered that 'private blockchains', e.g., Enterprise Blockchain can easily comply with GDPR directives since the transactions of the digital records of the stored infor- 


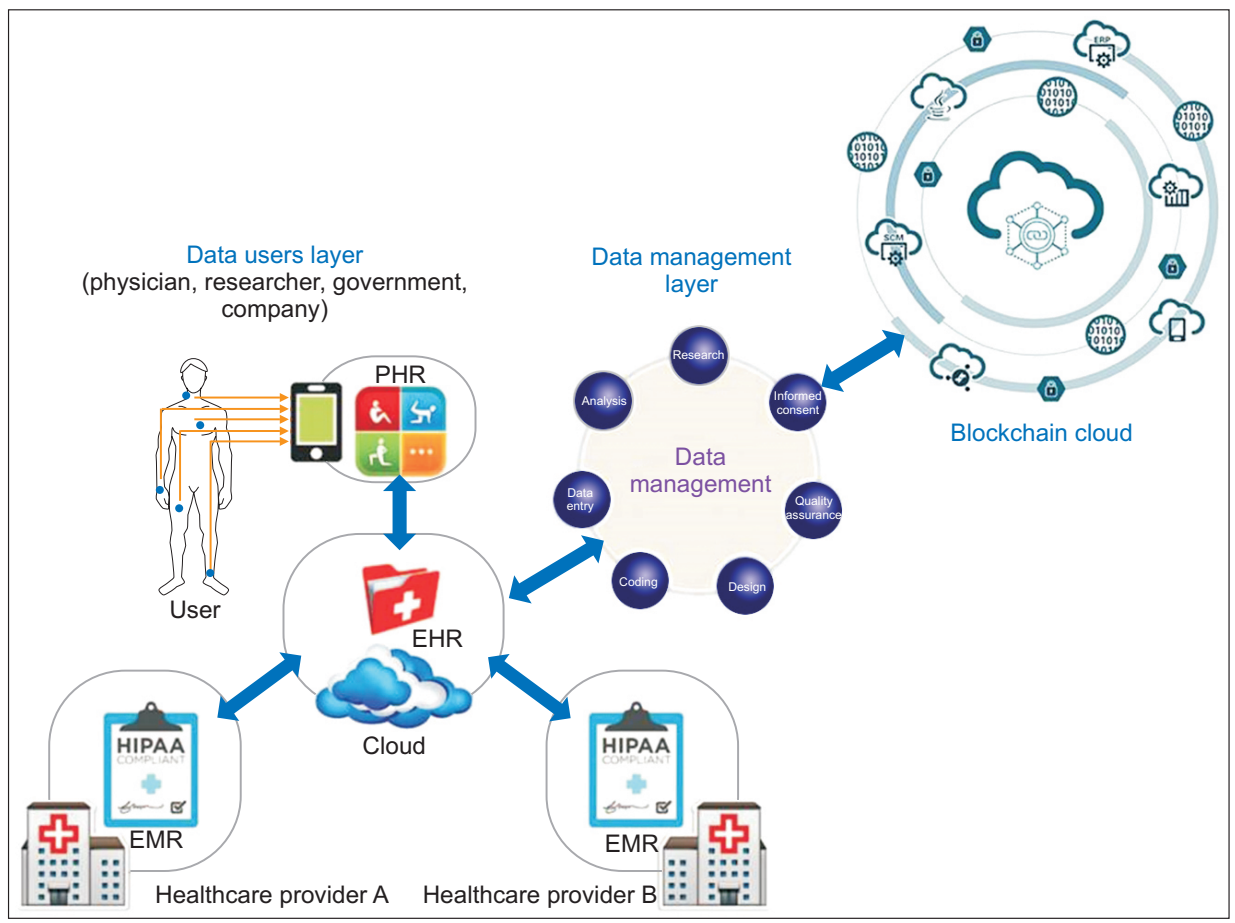

Figure 1. US pilot project 'Blockchain for Hospitals'. mation can be modified and erased by private entities or authorities who can own and control this platform, using a particular class of consensus algorithm [8]. These private blockchains are run by a single company or organization, but they grant access to users, typically organizations, that fulfill certain pre-established credentials or criteria [9]. Such systems would be similarly managed in terms of how a company manages its private web applications. Their use cases could include the following: record keeping by government agencies, public health records owners, and healthcare reimbursement providers. In the future, these private blockchains could have the most significant impact on healthcare policy and management. The potential of blockchains is also addressed by the European Commission Research \& Innovation Program IMI (Innovative Medicine Initiative) Pilot project named "Blockchain Enabled Healthcare" lead by Novartis, which aims to leverage existing standards, such as Ethereum, and to develop complementary standards if required. The focus is on enabling services that directly benefit patients [10].

\section{Blockchain for Personal Health Record (PHR) Data Management}

Personal life-log data recently has begun to be captured through wearable sensors or medical IoT devices as personal health records (PHR). Real-time artificial intelligence (AI)-powered healthcare analytics will be fed back to the related users, including patients, physicians, pharmaceutical researchers, and payers $[11,12]$. This entire PHR service trajectory is becoming a valuable source of data for blockchain service providers. (Figure 2).

Distributed or decentralized applications (Dapps) developed on the blockchain enable physicians and patients to easily participate in telemedicine with no middleman costs aside from the minimal fees of the Ethereum network, thus enhancing patient empowerment.

\section{Blockchain for Point-of-Care Genomics}

According to Timi Inc., which is a blockchain platform company, an individual patient's data is estimated to be worth as much as USD 7,000 per year [13]. Most of the mHealth companies developing blockchain services target the potential of patients to own and sell their health data with a focus on personal Electronic Health Records (EHRs) and wellnessroutine profiles gathered by wearable sensors, as well as the personal (at-home) genome [14]. Therefore, many consumer companies have been offering DNA sequencing for some time. The company ' 23 andMe', which was founded in 2006, is the most prolific, offering direct-to-consumer genetic testing services [15]. However, privacy is a major issue in the healthcare industry. Last year, 23andMe announced that they had sold a USD 300 million stake in the company to the pharmaceutical giant GlaxoSmithKline, effectively handing over access to the 5 million customers' data, despite it only containing exome data. To address such future concerns, blockchain startups in healthcare claim to offer a solution for 


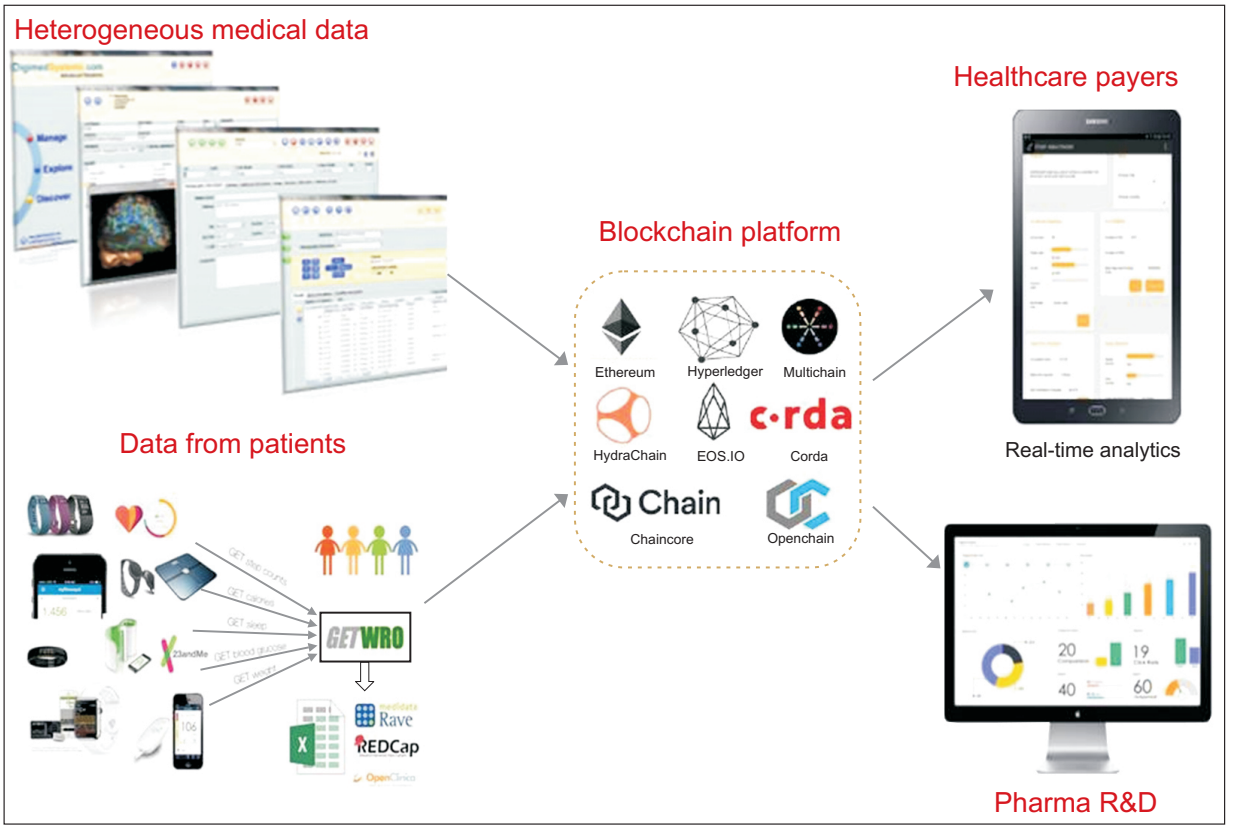

Figure 2. Personal health record data for blockchain service providers or data brokers. consumers wanting to have a DNA test done, while keeping data ownership. They offer the option of data monetization through blockchain-supported providers. For example, a startup called Nebula Genomics is offering whole-genome sequencing for free, as a way to stock up for its real ploy: a blockchain-based genetic marketplace [16]. Once users have their genome sequenced, they can charge a fee, in tokens, to anyone who wants to access it. Thereafter, those tokens can be redeemed for additional tests and products that will further interpret DNA. Genomes.io is another genomics blockchain company that allows consumers to securely store their genome from the moment it is sequenced and subsequently grant access selectively. The idea is to prevent genetic information getting into the wrong hands, while giving consumers the opportunity to sell their genetic data bit by bit if they wish to do so [17].

\section{Blockchain for EHR Data Management}

Blockchain technology is also a viable choice for personal EHR management. So-called 'smart contracts', could allow patients to be remunerated with tokens for their sharing of health data with providers and their research partners. For example, Health Wizz is piloting a blockchain- and FHIRenabled EHR aggregator mobile app, which uses blockchains to tokenize data, enabling patients to securely aggregate, organize, share, donate, and/or trade their personal medical records [18]. The idea is to enable individuals to control their health data as easily as they do their online bank accounts to allow better communication between healthcare organizations and caregivers to pave the way for a higher standard of care.

As an EHR blockchain company, Medicalchain is working on enabling various healthcare agents, such as doctors, hospitals, laboratories, pharmacists, and insurers, to request permission to access and interact with patients' medical records [19]. Each interaction is auditable, transparent, and secure and is recorded as a transaction on Medicalchain's distributed ledger.

\section{Discussion}

On a blockchain, it is cheap to verify the integrity of an individual transaction. A single piece of information can be audited in real time; moreover, its integrity is available to any participant in the network. As a result, costless verification can be economically implemented. For example, healthcare accounting information that can be built up with integrity from the simplest units of transactions has previously constituted a time consuming and costly audit. Now, with blockchains, this process can run continuously in the background in compliance with regulations [20].

There are five potential benefits of blockchains in comparison with traditional healthcare database management systems [5]. First, blockchains enable decentralized management; they are suitable for applications where healthcare stakeholders (e.g., hospitals, patients, payers, etc.) wish to collaborate with one another without the control of a central management intermediary. Second, blockchains provide immutable audit trails; they are suitable for unchangeable databases to record critical information (e.g., insurance claim re- 
cords). Third, blockchains enable data provenance; they are suitable for use in managing digital assets (e.g., patient consent in clinical trials). The ownership can only be changed by the owner, following cryptographic protocols. Also, the origins of the assets are traceable (i.e., the sources of the data and records can be confirmed), increasing the reusability of verified data. Fourth, blockchains ensure the robustness and availability of data; they are suitable for the preservation and continuous availability of records (e.g., the electronic health records of patients). Finally, they increase the security and privacy of data; data is encrypted in blockchains and can only be decrypted with the patient's private key. Even if the network is infiltrated by a malicious party, there is no practical way to read patient data.

\section{Conflict of Interest}

No potential conflict of interest relevant to this article was reported.

\section{Acknowledgments}

The Author would like to thank Mrs. Tajsa Zajc from Faces of Digital Health for the critical review of the Manuscript.

\section{References}

1. Wikipedia. Blockchain [Internet]. [place unknown]: Wikimedia Foundation; c2019 [cited at 2019 Jan 15]. Available from: https://en.wikipedia.org/wiki/Blockchain.

2. Wikipedia. InterPlanetary File System [Internet]. [place unknown]: Wikimedia Foundation; c2019 [cited at 2019 Jan 15]. Available from: https://en.wikipedia.org/wiki/ InterPlanetary_File_System.

3. Deloitte. Blockchain: opportunities for health care [Internet]. New York (NY): Deloitte; c2019 [cited at 2019 Jan 15]. Available from: https://www2.deloitte.com/us/ en/pages/public-sector/articles/blockchain-opportunities-for-health-care.html.

4. Gordon WJ, Catalini C. Blockchain technology for healthcare: facilitating the transition to patientdriven interoperability. Comput Struct Biotechnol J 2018;16:224-30.

5. Kuo TT, Kim HE, Ohno-Machado L. Blockchain distributed ledger technologies for biomedical and health care applications. J Am Med Inform Assoc 2017;24:1211-20.

6. Cyran MA. Blockchain as a foundation for sharing healthcare data. Blockchain Healthc Today 2018;1:13.
7. Kaye J, Whitley EA, Lund D, Morrison M, Teare H, Melham K. Dynamic consent: a patient interface for twentyfirst century research networks. Eur J Hum Genet 2015;23(2):141-6.

8. Lima C. Blockchain-GDPR privacy by design: how decentralized blockchain internet will comply with GDPR data privacy [Internet]. [place unknown: publisher unknown]; 2018 [cited at 2019 Jan 15]. Available from: https://blockchain.ieee.org/images/files/pdf/blockchaingdpr-privacy-by-design.pdf.

9. Commission Nationale de l'Informatique et des Libertes. Blockchain and the GDPR: solutions for a responsible use of the blockchain in the context of personal data [Internet]. Paris, France: Commission Nationale de l'Informatique et des Libertes; 2018 [cited at 2019 Jan 15]. Available from: https://www.cnil.fr/en/blockchainand-gdpr-solutions-responsible-use-blockchain-context-personal-data.

10. Innovative Medicines Initiative. Topic: Blockchain enabled healthcare [Internet]. Brussels, Belgium: Innovative Medicines Initiative; 2018 [cited at 2019 Jan 15]. Available from: https://www.imi.europa.eu/sites/default/ files/uploads/documents/apply-for-funding/futuretopics/Blockchain_vJune2018.pdf.

11. Dimitrov DV. Medical Internet of Things and big data in healthcare. Healthc Inform Res 2016;22(3):156-63.

12. Salah K, Rehman MH, Nizamuddin N, Al-Fuqaha A. Blockchain for AI: review and open research challenges. IEEE Access 2019;7:10127-49.

13. Timicoin [Internet]. [place unknown]: Timi Group Inc.; c2018 [cited at 2019 Jan 15]. Available from: https:// www.timicoin.io.

14. Zajc T. F020 Blockchain, value of data, and the role of legislation with adoption (Ray Dogum, Health Unchained) [Internet]. [place unknown: publisher unknown]; 2018 [cited at 2019 Jan 15]. Available from: https://medium.com/faces-of-digital-health/f020-blockchain-value-of-data-and-the-role-of-legislation-withadoption-ray-dogum-health-80919d909e97.

15. Bates M. Direct-to-consumer genetic testing: is the public ready for simple, at-home DNA tests to detect disease risk? IEEE Pulse 2018;9(6):11-14.

16. Nebula Genomics [Internet]. San Francisco (CA): Nebular Genomics; c2018 [cited at 2019 Jan 15]. Available from: http://www.nebula.org.

17. Genomes.io [Internet]. [place unknown]: Genomes. io; c2018 [cited at 2019 Jan 15]. Available from: https:// www.genomes.io. 
18. Health Wizz [Internet]. [place unknown]: Health Wizz; c2018 [cited at 2019 Jan 15]. Available from: https:// www.healthwizz.com.

19. Medicalchain [Internet]. [place unknown]: Medicalchain; c2018 [cited at 2019 Jan 15]. Available from: https://medicalchain.com/en/.

20. Catalini C, Gans JS. Some simple economics of the blockchain [Internet]. [place unknown]: SSRN; 2017 [cited at 2019 Jan 15]. Available from: http://dx.doi. org/10.2139/ssrn.2874598. 